\title{
Evidências acerca do uso de glicocorticoides como terapêutica para sepse: uma revisão integrativa de literatura
}

\author{
Evidence about the use of glucocorticoids as therapy for sepsis: an integrative literature review \\ Evidencia sobre el uso de glucocorticoides como terapia para la sepsis: una revisión integrativa de \\ la literatura
}

Recebido: 26/01/2022 | Revisado: 03/02/2022 | Aceito: 04/02/2022 | Publicado: 06/02/2022

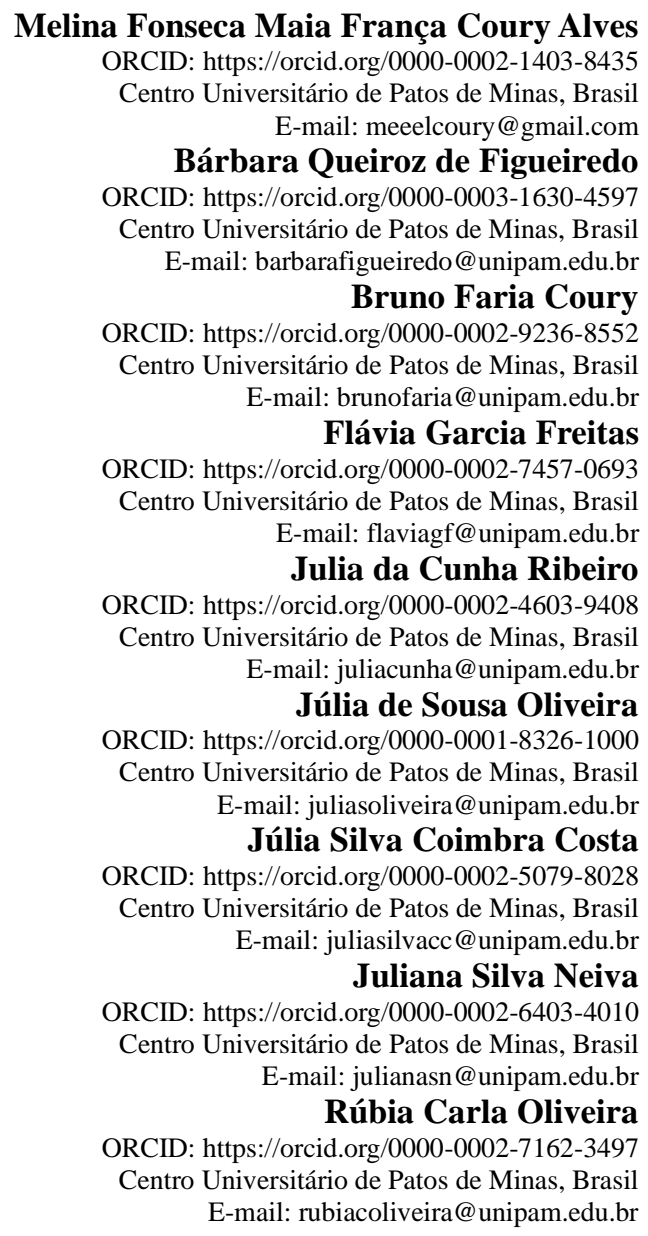

\begin{abstract}
Resumo
Introdução: a sepse consiste em uma doença inflamatória sistêmica associada a uma infecção com manifestações graves que ocorre por todo o organismo. No entanto, essa infecção não precisa estar necessariamente em todos os locais do organismo, pode estar restrita em apenas um local, mas causar uma resposta inflamatória por todo o corpo para tentar controlar a causa da infecção. Objetivo: demonstrar as atuais evidências sobre o uso de corticosteroides no tratamento da sepse em paciente hospitalizados nas unidades de terapia intensiva (UTI's). Metodologia: trata-se de uma pesquisa descritiva do tipo revisão integrativa da literatura. Foi realizado o cruzamento dos descritores, em inglês: "sepsis", "hemodynamic", "corticosteroids" em português: "sepse", "hemodinâmica", "corticosteroides" e em espanhol: "sepsis", "hemodinámico", "corticosteroides", nas seguintes bases de dados: National Library of Medicine (PubMed MEDLINE), Scientific Electronic Library Online (Scielo), Cochrane Database of Systematic Reviews (CDSR), Google Scholar, Biblioteca Virtual em Saúde (BVS) e EBSCO Information Services. Resultados e discussão: o tratamento pode ser feito com corticosteroides a fim de atenuar e modificar a resposta inflamatória, além de oferecerem um custo acessível. Porém, alguns efeitos colaterais e as formas adequadas de administração corroboram para que haja algumas controvérsias quanto ao uso dos corticoides, como a hidrocortisona, para tratamento de pacientes com sepse, mesmo com resultados satisfatórios. Considerações finais: os corticoides, em especial a
\end{abstract}


hidrocortisona, podem ser utilizados para o tratamento dos pacientes com sepse. Conforme as literaturas utilizadas, os resultados são satisfatórios e possuem um custeio de fácil adesão. Contudo, o uso destes medicamentos, mesmo apresentando benefícios, ainda é controverso, devido aos efeitos colaterais causados, à posologia e à forma de administração. Desta forma, discussões acerca deste assunto existem.

Palavras-chave: Glicocorticoides; Sepse; Terapia intensiva.

\begin{abstract}
Introduction: sepsis is a systemic inflammatory disease associated with an infection with severe manifestations that occurs throughout the body. However, this infection does not necessarily have to be everywhere in the body, it can be restricted to just one place, but cause an inflammatory response throughout the body to try to control the cause of the infection. Objective: to demonstrate the current evidence on the use of corticosteroids in the treatment of sepsis in patients hospitalized in intensive care units (ICUs). Methodology: this is a descriptive research of the integrative literature review type. The descriptors were crossed, in English: "sepsis", "hemodynamic", "corticosteroids" in Portuguese: "sepsis", "hemodynamics", "corticosteroids" and in Spanish: "sepsis", "hemodynamic", "corticosteroids", in the following databases: National Library of Medicine (PubMed MEDLINE), Scientific Electronic Library Online (Scielo), Cochrane Database of Systematic Reviews (CDSR), Google Scholar, Virtual Health Library (BVS) and EBSCO Information Services. Results and discussion: treatment can be done with corticosteroids in order to attenuate and modify the inflammatory response, in addition to offering an affordable cost. However, some side effects and adequate forms of administration corroborate the fact that there are some controversies regarding the use of corticosteroids, such as hydrocortisone, for the treatment of patients with sepsis, even with satisfactory results. Final considerations: corticosteroids, especially hydrocortisone, can be used to treat patients with sepsis. According to the literature used, the results are satisfactory and costing is easy to adhere to. However, the use of these drugs, even with benefits, is still controversial, due to the side effects caused, the dosage and the form of administration. Thus, discussions on this subject exist.
\end{abstract}

Keywords: Glucocorticoids; Sepsis; Intensive therapy.

\title{
Resumen
}

Introducción: la sepsis es una enfermedad inflamatoria sistémica asociada a una infección con manifestaciones graves que se presenta en todo el organismo. Sin embargo, esta infección no necesariamente tiene que estar en todas partes del cuerpo, puede estar restringida a un solo lugar, pero causar una respuesta inflamatoria en todo el cuerpo para tratar de controlar la causa de la infección. Objetivo: demostrar la evidencia actual sobre el uso de corticoides en el tratamiento de la sepsis en pacientes hospitalizados en unidades de cuidados intensivos (UCI). Metodología: se trata de una investigación descriptiva del tipo revisión integrativa de la literatura. Se cruzaron los descriptores, en inglés: "sepsis", "hemodynamic", "corticosteroids", en portugués: "sepsis", "hemodynamics", "corticosteroids" y en español: "sepsis", "hemodynamic", "corticosteroids", en las siguientes bases de datos: National Library of Medicine (PubMed MEDLINE), Scientific Electronic Library Online (Scielo), Cochrane Database of Systematic Reviews (CDSR), Google Scholar, Virtual Health Library (BVS) y EBSCO Information Services. Resultados y discusión: el tratamiento puede realizarse con corticoides para atenuar y modificar la respuesta inflamatoria, además de ofrecer un costo accesible. Sin embargo, algunos efectos secundarios y formas de administración adecuadas corroboran el hecho de que existen algunas controversias en cuanto al uso de corticoides, como la hidrocortisona, para el tratamiento de pacientes con sepsis, incluso con resultados satisfactorios. Consideraciones finales: los corticosteroides, especialmente la hidrocortisona, pueden usarse para tratar pacientes con sepsis. De acuerdo con la literatura utilizada, los resultados son satisfactorios y el costeo es fácil de cumplir. Sin embargo, el uso de estos fármacos, aun con beneficios, sigue siendo controvertido, debido a los efectos secundarios que provocan, la posología y la forma de administración. Por lo tanto, existen discusiones sobre este tema.

Palabras clave: Glucocorticoides; Septicemia; Terapia intensiva.

\section{Introdução}

A sepse consiste em uma doença inflamatória sistêmica associada a uma infecção com manifestações graves que ocorrem por todo o organismo. No entanto, essa infecção não precisa estar necessariamente em todos os locais do organismo, pode estar restrita em apenas um local, mas causar uma resposta inflamatória por todo o corpo para tentar controlar a causa da infecção (Souza et al., 2017; Neta et al, 2020). As respostas inflamatórias sistêmicas (SIRS) são caracterizadas pela Society of Critical Care of Medicine seguindo alguns critérios, tais como: febre ou hipotermia; frequência cardíaca maior que 90 bpm; taquipneia ou $\mathrm{PaCO}_{2}<32 \mathrm{mmHg}$, ou leucocitose $(>12.000)$, leucopenia $(<4.000)$ ou presença de formas imaturas no leucograma (> 10\%) (de Mello et al., 2011). Nesse sentido, é importante ressaltar que sepse e choque séptico são condições diferentes. Na sepse pode ocorrer o comprometimento de vários órgãos do paciente, causando falência múltipla. Além disso, 
no quadro da sepse os macrófagos são estimulados por meio de várias vias de ativação da inflamação, sendo esse um dos motivos pelo qual o tratamento com corticosteroides é administrado nesse quadro. Já o choque séptico é uma forma mais grave da sepse, a inflamação alcança um estágio avançado e não possui respostas quanto ao tratamento de fluidos (Neta et al., 2020).

Quanto à epidemiologia, a sepse é uma condição patológica extremamente frequente, que representa uma das principais causas de óbitos e hospitalização nas unidades de terapia intensiva (UTI's). Estima-se que a sepse tenha uma incidência mundial de 31,5 milhões de casos por ano, com cerca de 5,3 milhões de óbitos (Souza et al., 2017). Em adição, Taniguchi et al. (2019) realizaram um estudo nas UTI's brasileiras e verificaram que algumas unidades não possuem suporte adequado para o tratamento de pacientes sépticos ou até mesmo procedimentos básicos para monitoramento são escassos, o que contribui para uma maior taxa de mortalidade

Diante disso, os corticosteroides conhecidos como agentes anti-inflamatórios e imunossupressores, são utilizados no tratamento da sepse com o objetivo de atenuar e modificar a resposta inflamatória. Esses medicamentos exercem os seus efeitos por meio da modulação da transcrição de genes inflamatórios e da imunossupressão. As células nucleadas expressam alguns receptores intracelulares de corticoides, estes, por sua vez, ligam-se aos corticoides formando um complexo esteroidereceptor que ao penetrar no núcleo das células alvo modula a transcrição de genes que codificam proteínas anti-inflamatórias (Ramanan et al., 2019).

Além disso, este medicamento atua inibindo o fator nucelar kappa beta (NF-kB) e a síntese de citocinas inflamatórias como o fator de necrose tumoral alfa (TNF- $\alpha$ ), interleucina 1 (IL-1) e interleucina 6 (IL-6). Outra ação dos corticosteroides consiste na diminuição da migração de células inflamatórias para os locais de lesão ou inflamação, o que reduz a expressão de moléculas de adesão endotelial e de adesão intracelular, de prostaglandinas e de quimiocinas. Tais efeitos permite atenuar a resposta inflamatória do paciente. (Salluh et al., 2017). Ademais, Ramanan et al. (2019) afirmam ainda que as evidências quanto às dosagens de corticosteroides sugerem o uso de hidrocortisona com uma dose de $200 \mathrm{mg}$ por dia durante 7 dias. Os autores relataram, também, que uma dose de 300mg em comparação com $200 \mathrm{mg}$ não apresenta muito benefícios, além de que pode aumentar a taxa de recorrência da sepse após a cessação do medicamento. Diante disso, a hidrocortisona possui vários benefícios em relação aos recursos de saúde, tais como: é uma droga barata e segura, possibilita menor permanência na UTI, menor tempo de ventilação mecânica e resolução mais rápida da sepse. Deste modo, este estudo teve por objetivo realizar uma revisão bibliográfica a fim de apresentar as evidências atuais sobre o uso de corticoides no tratamento da sepse em paciente hospitalizados nas unidades de terapia intensiva (UTI's).

\section{Metodologia}

Trata-se de uma pesquisa descritiva do tipo revisão integrativa da literatura. Para a elaboração da questão de pesquisa da revisão integrativa utilizou a estratégia PICOT (Acrômio para Patient, Intervention, Comparation, Outcome and Time). O uso dessa estratégia para formular a questão de pesquisa na condução de métodos de revisão possibilita a identificação de palavras-chave, as quais auxiliam na localização de estudos primários relevantes nas bases de dados. Assim, a questão de pesquisa delimitada foi: "quais as evidências sobre o uso de corticosteroides no tratamento de sepse?". Dessa maneira, compreende-se que P= Indivíduos sob sepse; I = Indivíduos que utilizaram corticosteroides como intervenção para a sepse; Co = Indivíduos em tratamento para sepse sem utilização de corticosteroides; $\mathrm{T}=$ Duas semanas de coleta de dados. A partir do estabelecimento das palavras-chave da pesquisa, foi realizado o cruzamento dos descritores, em inglês: "sepsis", "hemodynamic", "corticosteroids" em português: "sepse", "hemodinâmica", "corticosteroides" e em espanhol: "sepsis", "hemodinámico", "corticosteroides", nas seguintes bases de dados: National Library of Medicine (PubMed MEDLINE), Scientific Electronic Library Online (Scielo), Cochrane Database of Systematic Reviews (CDSR), Google Scholar, Biblioteca Virtual em Saúde (BVS) e EBSCO Information Services. 
A pesquisa bibliográfica foi de cunho exploratório, partindo da identificação, da seleção e da avaliação de trabalhos e de artigos científicos considerados relevantes para dar suporte teórico para a classificação, a descrição e a análise dos resultados. A busca foi realizada nos meses de setembro e outubro de 2021. Foram considerados estudos publicados no período compreendido entre 2011 e 2021. A estratégia de seleção dos artigos seguiu as seguintes etapas: busca nas bases de dados selecionadas; leitura dos títulos de todos os artigos encontrados e exclusão daqueles que não abordavam o assunto; leitura crítica dos resumos dos artigos e leitura na íntegra dos artigos selecionados nas etapas anteriores.

Foram analisadas fontes relevantes inerentes ao tema, utilizando como um dos principais critérios a escolha de artigos atuais, originais e internacionais. Após leitura criteriosa das publicações, 3 artigos não foram utilizados devido aos critérios de exclusão. Assim, totalizaram-se 24 artigos científicos para a revisão integrativa da literatura, com os descritores apresentados acima. Após esta seleção, filtraram-se por artigos dos últimos dez anos e por artigos em línguas portuguesa, espanhola e inglesa. Como critérios de inclusão, foram considerados artigos originais, que abordassem o tema pesquisado e permitissem acesso integral ao conteúdo do estudo, no período de 2011 a 2021, em inglês, espanhol e português. O critério de exclusão foi imposto naqueles trabalhos que não estavam em espanhol, inglês ou português, que não tinham passado por processo de PeerView e que não relacionasse a utilização de corticosteroides para o tratamento de sepse.

\section{Resultados e Discussão}

Realizado o levantamento bibliográfico seguindo os critérios de inclusão e exclusão, os periódicos selecionados foram agrupados no Quadro 1 e classificados de acordo com autoria/ano, tipo de estudo e principais resultados de cada obra.

Quadro 1: Distribuição dos artigos conforme autoria/ano, título, objetivo e resultado.

\begin{tabular}{|c|c|c|}
\hline $\begin{array}{c}\text { AUTOR E } \\
\text { ANO }\end{array}$ & $\begin{array}{l}\text { TIPO DE } \\
\text { ESTUDO }\end{array}$ & PRINCIPAIS ACHADOS \\
\hline $\begin{array}{c}\text { de Mello et al., } \\
2011\end{array}$ & $\begin{array}{c}\text { Revisão narrativa de } \\
\text { literatura }\end{array}$ & $\begin{array}{l}\text { Recomenda iniciar hidrocortisona ( } 200 \text { a } 300 \mathrm{mg} / \mathrm{dia}) \text {, em infusão contínua ou em doses fracionadas em } 4 \\
\text { vezes ao dia, naqueles pacientes em uso de doses altas de vasopressores, a despeito da reposição volêmica; e } \\
\text { doses superiores não estão indicadas. }\end{array}$ \\
\hline $\begin{array}{c}\text { Ramanan et al., } \\
2019\end{array}$ & $\begin{array}{c}\text { Revisão sistemática } \\
\text { de literatura }\end{array}$ & $\begin{array}{l}\text { A hidrocortisona é uma droga relativamente barata e segura. Não possui benefício com relação a mortalidade } \\
\text { em choque séptico, porém quanto a utilização de recursos de saúde: menor permanência na UTI, menor tempo } \\
\text { de ventilação mecânica e resolução mais rápida do choque, o que gera economia, já que um leito de UTI pode } \\
\text { custar milhares de dólares por dia, enquanto um curso de } 7 \text { dias de hidrocortisona custa }<\$ 150 \text {. }\end{array}$ \\
\hline $\begin{array}{l}\text { Fang et al., } \\
2019\end{array}$ & Metanálise & $\begin{array}{l}\text { O tratamento com corticosteroides foi associado com a redução da mortalidade em } 28 \text { dias, mortalidade na UTI } \\
\text { e morbilidade hospitalar entre pacientes com sepse, benefício de sobrevivência que não foi replicado com a } \\
\text { mortalidade de } 90 \text { dias. Aumento significativo na reversão do choque e dias sem vasopressores até o dia } 28 \text { e } \\
\text { com uma diminuição acentuada no tempo de permanência na UTI, pontuação SOFA aos } 7 \text { dias, e tempo para a } \\
\text { resolução do choque. A administração de corticosteroides estava associada a um risco aumentado de } \\
\text { hipernatremia e hiperglicemia. }\end{array}$ \\
\hline $\begin{array}{c}\text { Taniguchi et al., } \\
2014\end{array}$ & Metanálise & $\begin{array}{l}\text { Uma porcentagem importante de unidades não tem condições para realizar intervenções básicas de } \\
\text { monitoramento e terapêutica em pacientes sépticos, mesmo a sepse sendo uma prioridade global de saúde, } \\
\text { conforme declarado pela Organização Mundial da Saúde. Com base em recente revisão sistemática, é estimada } \\
\text { a incidência mundial de } 31,5 \text { milhões de casos de sepse ao ano, com potencial de } 5,3 \text { milhões de óbitos, } \\
\text { baseado em dados de países com níveis elevados de renda. Considerando que mais de } 80 \% \text { da população } \\
\text { mundial vive em países com baixa e média renda (PBMRs), nos quais são frequentes as limitações de recursos, } \\
\text { as taxas de letalidade reais são, possivelmente, muito mais elevadas. }\end{array}$ \\
\hline $\begin{array}{c}\text { Lamontagne et } \\
\text { al., } 2018\end{array}$ & $\begin{array}{c}\text { Revisão sistemática } \\
\text { de literatura }\end{array}$ & $\begin{array}{l}\text { Conclui-se que é possível que os corticosteroides ajudem na melhora da resposta imunológica desregulada } \\
\text { causada pela sepse e aumentar a pressão arterial quando baixa. A maioria das organizações profissionais não } \\
\text { recomenda o uso de corticosteroides na ausência de choque refratário. Adicionar um agente com atividade } \\
\text { mineralocorticoide adicional, como a fludrocortisona, pode ser útil, mas isso é altamente especulativo. }\end{array}$ \\
\hline $\begin{array}{c}\text { Tayaré et al., } \\
2017\end{array}$ & $\begin{array}{l}\text { Diretriz clínica de } \\
\text { recomendação }\end{array}$ & $\begin{array}{l}\text { É recomendada que a avaliação inicial de pacientes com suspeita de infecção deve incluir a identificação da } \\
\text { fonte, quaisquer fatores que tornariam o paciente mais suscetível à sepse e indicadores de preocupação clínica, } \\
\text { em particular indicações de problemas mentais estado, circulação ou respiração. Os antibióticos não devem ser } \\
\text { administrados, a menos que haja suspeita específica de doença meningocócica (erupção cutânea que não } \\
\text { empalidece na presença de febre). }\end{array}$ \\
\hline Lin, 2020 & $\begin{array}{c}\text { Revisão integrativa } \\
\text { de literatura }\end{array}$ & $\begin{array}{l}\text { Foram expostos estudos contrários ao uso de altas doses de metilprednisolona em pacientes com COVID-19 e } \\
\text { sepse, já que nenhum benefício significativo foi encontrado na prevenção do choque, reversão do choque e } \\
\text { diminuição da mortalidade. Também foi relatada uma metanálise da década de } 1990 \text {, que relata o tratamento da } \\
\text { sepse com corticosteroides indicando para o aumento da mortalidade e sangramento gastrointestinal. }\end{array}$ \\
\hline Fuji et al., 2020 & $\begin{array}{c}\text { Revisão integrativa } \\
\text { de literatura }\end{array}$ & $\begin{array}{l}\text { Os ensaios abordados nessa revisão indicaram que a administração de baixas doses de corticosteroides } \\
\text { resultaram na diminuição da duração do choque. Um ensaio abordando a eficácia da vitamina C no tratamento } \\
\text { da sepse, sem que houvessem resultados com alterações significativas sobre disfunções orgânicas ou }\end{array}$ \\
\hline
\end{tabular}




\begin{tabular}{|c|c|c|}
\hline & & $\begin{array}{l}\text { biomarcadores. Ensaios sobre a administração de tiamina no tratamento da sepse demonstraram um resultado } \\
\text { neutro. A combinação das três terapias citadas acima, feita em um ensaio, descobriu que a mesma não } \\
\text { aumentava o tempo livre de vasopressores. Dois ensaios aleatórios sobre a suplementação de vitamina D nos } \\
\text { doentes críticos não conseguiram identificar uma redução estatisticamente significativa na mortalidade. Nessa } \\
\text { revisão, não foram encontradas provas suficientes que comprovem a eficiência das terapias citadas, sendo } \\
\text { necessários dados de estudos bem concebidos sobre a administração dos compostos. }\end{array}$ \\
\hline $\begin{array}{l}\text { Rygård et al., } \\
2020\end{array}$ & $\begin{array}{l}\text { Ensaio clínico } \\
\text { randomizado }\end{array}$ & $\begin{array}{l}\text { Em adultos com choque séptico tratados com corticosteroides em baixas doses, a mortalidade a curto e longo } \\
\text { prazo não é afetada, os eventos adversos aumentam, mas a duração do choque, a ventilação mecânica e a } \\
\text { estadia na UCI são reduzidas. }\end{array}$ \\
\hline $\begin{array}{l}\text { Moskowitz et } \\
\text { al., } 2020\end{array}$ & $\begin{array}{l}\text { clínico } \\
\text { nizado }\end{array}$ & $\begin{array}{l}\text { A combinação de ácido ascórbico, corticosteroides e tiamina, em comparação com placebo, não resultou numa } \\
\text { redução estatisticamente significativa, em pacientes com choque séptico, nos escores SOFA durante as } \\
\text { primeiras } 72 \text { horas após a inscrição. Estes dados não suportam utilização rotineira desta terapia combinada para } \\
\text { pacientes com choque séptico. }\end{array}$ \\
\hline $\begin{array}{l}\text { Vandewalle et } \\
\text { al., } 2020\end{array}$ & $\begin{array}{l}\text { Revisão integrativa } \\
\text { de literatura }\end{array}$ & $\begin{array}{l}\text { O uso de corticosteroides no tratamento da sepse continua a ser assunto de debates. Estudos realizados para a } \\
\text { avaliaçãa da eficácia dos Glicocorticoides, da vitamina C e da tiamina como monoterapia, demonstraram a } \\
\text { presença de todos os três efeitos protetores, mas limitados no manejo do choque séptico. Já em estudos em que } \\
\text { essas três terapias foram combinadas, foi possível observar a redução da mortalidade hospitalar de } 40,4 \% \text { para } \\
8,5 \% \text { nos pacientes com sepse, sem que houvesse aumento dos efeitos adversos. }\end{array}$ \\
\hline $\begin{array}{l}\text { Salluh et al., } \\
\quad 2017\end{array}$ & $\begin{array}{r}\text { Revisão } \\
\text { de li }\end{array}$ & $\begin{array}{l}\text { Em algumas situações clínicas relevantes, como na pneumonia pneumocystis jirovecii e meningite } \\
\text { pneumocócica o uso de corticosteroides é apoiado por fortes evidências, em sepse grave ou choque séptico, } \\
\text { atualmente, não está associado a melhores desfechos, portanto seu uso estabelecido em pacientes com choque } \\
\text { séptico não é recomendado. }\end{array}$ \\
\hline $\begin{array}{l}\text { Heming et al., } \\
2020\end{array}$ & $\begin{array}{r}\text { Revisão } \\
\text { de li }\end{array}$ & $\begin{array}{l}\text { Os tratamentos considerados de primeira linha incluem a administração precoce da terapia antimicrobiana } \\
\text { apropriada e controle de origem, associada ao apoio aos órgãos. O manejo hemodinâmico da sepse depende } \\
\text { da administração de fluidos e vasopressores, guiados pela avaliação dinâmica dos índices cardiovasculares } \\
\text { e dos níveis de lactato. }\end{array}$ \\
\hline Lu et al., 2020 & Estudo de coorte & $\begin{array}{l}\text { A terapia de corticosteroides para a manutenção da pressão arterial não foi associada à melhora da } \\
\text { mortalidade ou estabilidade hemodinâmica na população imunocomprometida com choque séptico. }\end{array}$ \\
\hline Shi et al., 2020 & Metanálise & $\begin{array}{l}\text { A corticoterapia pode restaurar o sistema imunológico desregulado, a função mitocondrial oxidativa e a } \\
\text { produção de energia, além do baixo risco, baixo custo e fácil disponibilidade. Foi constatado que pequenos } \\
\text { efeitos colaterais clínicos, como hiperglicemia, hipertensão e hipernatremia induzida por hidrocortisona, podem } \\
\text { ocorrer, mas são insignificantes e facilmente gerenciados na UTI. }\end{array}$ \\
\hline Neta et al., 2020 & $\begin{array}{l}\text { Revisão narrativa de } \\
\text { literatura }\end{array}$ & $\begin{array}{l}\text { Efeitos benéficos analisados na prática clínica: reversão do choque séptico em menor tempo, melhora das } \\
\text { variáveis hemodinâmicas e estabilidade do paciente. No entanto, não reduz significativamente a mortalidade e } \\
\text { há relatos de potenciais efeitos adversos, incluindo superinfecção e efeitos metabólicos e neuromusculares, } \\
\text { agravando a incerteza clínica. A administração dos corticosteroides tem se justificado para modulação do } \\
\text { processo inflamatório através da inibição da ativação de NF-kB, capaz de regular a expressão de muitos genes } \\
\text { inflamatórios e imunológicos. }\end{array}$ \\
\hline $\begin{array}{l}\text { Montes et al., } \\
\quad 2017\end{array}$ & $\begin{array}{l}\text { Revisão integrativa } \\
\text { de literatura }\end{array}$ & $\begin{array}{l}\text { Primordialmente favorável ao uso de corticosteroides por seu papel na diminuição das citocinas inflamatórias, } \\
\text { atenuando a disfunção orgânica e melhorando a hemodinâmica. Há menções de que pacientes em estado de } \\
\text { insuficiência adrenal, hipercortisolismo sem resposta ao teste da corticotrofina no momento da sepse tinham o } \\
\text { pior desfecho e seriam os mais beneficiados da corticoterapia. Além disso, não foi constatado benefício } \\
\text { expressivo na faixa etária pediátrica pelo risco de efeitos adversos frente à resposta controversa. }\end{array}$ \\
\hline $\begin{array}{l}\text { Lobo et al., } \\
2019\end{array}$ & $\begin{array}{c}\text { Revisão integrativa } \\
\text { de literatura }\end{array}$ & $\begin{array}{l}\text { Embora existam declínios progressivos nas taxas de mortalidade, as diferenças entre os hospitais são } \\
\text { preocupantes e os esforços das políticas de saúde pública devem se concentrar em melhorias de gestão. } \\
\text { Principalmente nas UTI’s públicas, a presença de recursos, entre eles medicamentosos, deve ser viabilizada. }\end{array}$ \\
\hline $\begin{array}{l}\text { Souza et al., } \\
2017\end{array}$ & $\begin{array}{c}\text { Revisão narrativa de } \\
\text { literatura }\end{array}$ & $\begin{array}{l}\text { A assistência hospitalar desenvolvida para o combate a sepse, não apresenta uma intervenção efetiva na } \\
\text { letalidade da doença; os casos devem ser detectados o mais precoce possível, pois a prevençá é forma mais } \\
\text { eficaz de combate à doença. }\end{array}$ \\
\hline Yao et al., 2019 & $\begin{array}{c}\text { Revisão sistemática } \\
\text { de literatura }\end{array}$ & $\begin{array}{l}\text { A terapia aditiva em pacientes que sofrem de sepse e choque séptico pelo uso de glicocorticoides atenua a } \\
\text { resposta pro-inflamatória, limita a resposta anti-inflamatória e preserva a imunidade inata. Além disso, os } \\
\text { glicocorticoides exógenos estão amplamente disponíveis, possuem baixo custo e capacidade para inibir a } \\
\text { cascata inflamatória }\end{array}$ \\
\hline $\begin{array}{l}\text { Tagami et al., } \\
2014\end{array}$ & $\begin{array}{l}\text { Revisão narrativa de } \\
\text { literatura }\end{array}$ & $\begin{array}{l}\text { Os resultados indicam que pode haver uma associação significativa entre o uso de corticosteroides de baixa } \\
\text { dose e a mortalidade de } 28 \text { dias em pacientes com PAC grave e choque. Foi avaliado o uso precoce e } \\
\text { prolongado de corticosteroides de baixa dose, pois os resultados de estudos anteriores sugeriram que o uso de } \\
\text { corticosteroide de curta duração, tardia e alta dose não são benéficos em pacientes com PAC grave. Apesar } \\
\text { disso, essa associação não foi encontrada em pacientes com PAC grave sem choque }\end{array}$ \\
\hline
\end{tabular}

Fonte: Autores (2021).

Assim, haja vista que a sepse é caracterizada como uma resposta inflamatória sistêmica mediada por citocinas e é responsável por altas taxas de morbimortalidade, o uso dos corticosteroides como terapia medicamentosa pode ser adequado para o manejo da sepse, pois bloqueia processos para a síntese e a circulação de mediadores inflamatórios (de Mello et al., 2011). A hidrocortisona em baixas doses é recomendada pelo Surviving Sepsis Campaign e é uma droga relativamente barata e segura. A administração pode reduzir o período de permanência dos pacientes na UTI, além de um menor período na ventilação mecânica e uma resolução rápida do choque. Entretanto, o uso da hidrocortisona ainda é controverso, uma vez que existem dúvidas sobre a sua dose, duração e método de aplicação (Ellender et al., 2020). 
Os glicocorticoides apresentaram potencial ação anti-inflamatória, além de estarem relacionados a melhorias no sistema cardiovascular, com potencial reversão hemodinâmica, além de estabilizarem o paciente com sepse grave (Lian et al., 2019). Outrossim, os glicocorticoides atenuam a resposta pró-inflamatória, limitam a resposta anti-inflamatória e preservam a imunidade inata, sendo eficaz a sua administração como uma terapia aditiva em pacientes com sepse e choque séptico (Yao et al., 2019). Os resultados analisados, em sua grande parte, evidenciaram benefícios no uso dos corticosteroides em pacientes com sepse, apesar da presença de efeitos colaterais pouco relevantes, como hiperglicemia, hipertensão e hipernatremia, que são de fácil controle na UTI (Rocha et al., 2011). O papel do uso dos corticosteroides na redução da mortalidade em pacientes com sepse ainda permanece pouco esclarecido e, somado a questões abordadas como seus efeitos adversos e formas adequadas de administração ainda contribuem com o debate acerca do assunto (Ramanan et al., 2019).

\section{Considerações Finais}

Neste sentido, conclui-se que os corticoides, em especial a hidrocortisona, podem ser utilizados para o tratamento dos pacientes com sepse. Conforme as literaturas utilizadas, os resultados são satisfatórios e possuem um custeio de fácil adesão. Contudo, o uso destes medicamentos, mesmo apresentando benefícios, ainda é controverso, devido aos efeitos colaterais causados, à posologia e à forma de administração. Desta forma, discussões acerca deste assunto existem.

\section{Referências}

de Mello, A. C. P. M., et al. (2011). Corticoterapia no choque séptico e sepse grave. Revista da Sociedade Brasileira de Terapia Intensiva, 9 (1), $50-53$.

Ellender, T., et al. (2020). Updates in Sepsis Resuscitation. Emergency Medicine Clinics of North America, 38 (4), $807-818$.

Fang, D., et al. (2019). Association of corticosteroid treatment with outcomes in adult patients with sepsis: a systematic review and meta-analysis. JAMA Internal Medicine, 179 (2), 213-223.

Fuji, T., et al. (2020). Metabolic support in sepsis: corticosteroids and vitamins: the why, the when, the how. Curr. Opin. Crit. Care, 26(4), 363-368.

Heming, N., et al. (2020). Sepsis in the critically ill patient: current and emerging management strategies. Expert Review of Anti-Infective Therapy, 7 (8), 111.

Lamontagne, F., et al. (2018). Corticosteroid therapy for sepsis: a clinical practice guideline. BMJ, 9 (7), 1-14.

Lian, X. J., et al. (2019). Reevaluating the Role of Corticosteroids in Septic Shock: An Updated Meta-Analysis of Randomized Controlled Trials. Biomed Res Int., 1 (8), 56-70.

Lin, H. Y. (2020). The severe COVID-19: a sepsis induced by viral infection? And its immunomodulatory therapy. Chinese Journal of Traumatology, 9 (6), $39-45$.

Lu, X., et al. (2020). Efficacy and safety of corticosteroids for septic shock in immunocompromised patients: A cohort study from MIMIC. The American Journal of Emergency Medicine, 5 (8), 1-9.

Lobo, S. M., et al. (2019). Mortalidade por sepse no Brasil em um cenário real: o projeto UTIs brasileiras. Revista Brasileira de Terapia Intensiva, 4 (1), 3444.

Montes, P. S., et al. (2017). Uso de corticosteroides em pacientes com sepse: uma revisão bibliográfica. Revista Brasileira de Terapia Intensiva, 4 (8), 1-8.

Moskowitz, A. et al. (2020). Effect of Ascorbic Acid, Corticosteroids, and Thiamine on Organ Injury in Septic Shock: The ACTS Randomized Clinical Trial. JAMA, 324 (7), 642-650.

Neta, A. B. C. S., et al. (2020). Relações da corticoterapia no tratamento do choque séptico. Brazilian Journal of Health Review, 3 (1), $1324-1330$.

Ramanan, M., et al. (2019). Steroids and Sepsis: The Debate Continues. International Anesthesiology Clinics, 57 (2), $17-30$.

Rocha, S. L., et al. (2011). Resposta hemodinâmica após uso de corticoide na sepse experimental. Revista do Médico Residente, 13 (1), 1-11.

Rygård, S. L. et al. (2018). Low-dose corticosteroids for adult patients with septic shock: a systematic review with meta-analysis and trial sequential analysis. Intensive Care Med, 44 (7), 1003-1016.

Salluh, J., et al. (2017). Corticosteroids in Severe Sepsis and Septic Shock. Shock, 47 (1), 47-51.

Shi, R., et al. (2020). Benefit of hydrocortisone, thiamine, and vitamin C for patients with sepsis or septic shock? Too early to draw conclusions. Critical Care, 24(1), 18-28. 
Research, Society and Development, v. 11, n. 2, e59211226196, 2022

(CC BY 4.0) | ISSN 2525-3409 | DOI: http://dx.doi.org/10.33448/rsd-v11i2.26196

Souza, C. N. S., et al. (2017). Sepse em ambiente hospitalar. Brazilian Journal of Health Review, 1 (7), 15-24.

Tagami, T. et al. (2014). Low-dose corticosteroid use and mortality in severe communityacquired pneumonia patients The European Respiratory Journal, 45 (2), 463-472.

Taniguchi, L. U., et al. (2019). Disponibilidade de recursos para tratamento da sepse no Brasil: uma amostra aleatória de instituições brasileiras. Revista Brasileira de Terapia Intensiva, 31 (2), 193-201.

Tavaré A. et al. (2017). Recognition, diagnosis, and early management of sepsis: NICE guideline. Br J Gen Pract, 67 (1), $185-166$.

Vandewalle, J., et al. (2020). Glucocorticoids in Sepsis: To Be or Not to Be. Front. Immunol., 11 (13), 1-11.

Yao, Y., et al. (2019). Are corticosteroids beneficial for sepsis and septic shock? based on pooling analysis of 16 studies. Frontiers in pharmacology, 10 (8), 714-718. 